\title{
Application of thrombolysis in myocardial infarction risk index in the prediction of long-term outcomes for patients with ST-elevation myocardial infarction and multiple vessel disease: A single-center prospective observational cohort study
}

\author{
XUEFANG FAN $^{1 *}$, MINGLIANG LI $^{2 *}$, JIE CAO $^{3}$ and ZEMING LIANG ${ }^{4}$ \\ ${ }^{1}$ Department of Cardiology, Xianyang Hospital of Yan'an University, Xianyang, Shanxi 712000; \\ ${ }^{2}$ Ward No. 2, Department of Cardiovascular Disease, People's Hospital of Hanzhong City, Hanzhong, \\ Shanxi 723000; ${ }^{3}$ Department of Cardiology, Affiliated Hospital of Yan'an University, \\ Xiangyang, Shanxi 716000; ${ }^{4}$ Second Department of Cardiovascular Disease, \\ Baoji Hospital of Traditional Chinese Medicine, Baoji, Shanxi 721000, P.R. China
}

Received July 23, 2019; Accepted November 21, 2019

DOI: $10.3892 /$ etm.2021.10899

\begin{abstract}
The thrombolysis in myocardial infarction (TIMI) risk index has been indicated to be a simple and useful tool for risk stratification of patients with ST-elevation myocardial infarction (STEMI). However, the predictive value of the TIMI risk index regarding the long-term outcome for patients with STEMI with multiple vessel disease has remained to be determined. In the present study, a total of 369 patients diagnosed with STEMI who received emergency percutaneous coronary intervention treatment were analyzed. A five-year follow-up was performed to record the primary endpoint of all-cause mortality, as well as the secondary endpoints of myocardial infarction, stroke, emergent revascularization and admission due to heart failure. A receiver operating characteristic (ROC) curve was used to determine the cut-off value of the TIMI risk index for predicting all-cause death, based on which the patients were divided into a high TIMI group and a low TIMI group. Kaplan-Meier survival curves were used to compare the long-term survival of the two groups and multivariate Cox regression analysis was used to evaluate the predictive value of the risk factors regarding primary and secondary endpoints. The ROC curve indicated that the TIMI risk index was associated with three-year all-cause death with a cut-off
\end{abstract}

Correspondence to: Dr Zeming Liang, Second Department of Cardiovascular Disease, Baoji Hospital of Traditional Chinese Medicine, 43 Baofu Road, Baoji, Shanxi 721000, P.R. China

E-mail: bjzyxnk@163.com

${ }^{*}$ Contributed equally

Key words: thrombolysis in myocardial infarction, ST-elevation myocardial infarction, all-cause death, cardiac death, stent thrombosis, long-term outcomes value of 30.35 (area under curve, $0.705 ; \mathrm{P}=0.001$ ). The high TIMI group (>30.35) and low TIMI group $(<30.35)$ exhibited a significant difference in all-cause death $(\mathrm{P}=0.009)$ but not in any of the secondary endpoints $(\mathrm{P}=0.527)$. Multivariate Cox regression analysis demonstrated that a high TIMI risk index was an independent risk factor for all-cause death in patients with STEMI and multiple-vessel disease (hazard ratio=3.709, 95\% CI: 1.521-9.046, $\mathrm{P}=0.004)$. In conclusion, the TIMI risk index was associated with long-term outcomes for patients with STEMI and multiple-vessel disease and may be of value for risk prediction.

\section{Introduction}

ST-segment elevation myocardial infarction (STEMI) remains the most dangerous type of acute coronary syndrome (ACS), causing $>30 \%$ of all mortalities and significantly increasing the familial and social health burden (1). Particularly patients with multiple vessel disease have a higher mortality and morbidity (2). Long-term outcomes of STEMI are associated with complex factors, including demographics, comorbidities and severity of STEMI. Various tools have been developed (3) and biomarkers have been used $(4,5)$ for predicting outcomes for patients with STEMI. Risk stratification helps to identify high-risk patients and apply prevention measures. However, those tools have certain disadvantages, including unstable performance, complex calculation and low predictive value. Sakamoto et al (6) compared the HEART, TIMI and GRACE scores for the prediction of 30-day major adverse cardiac events in patients with high acuity chest pain in the emergency department and found that the HEART score to be superior to the TIMI and GRACE scores in predicting 30-day MACE. In addition, Hammami et al (7) demonstrated that the GRACE and TIMI scores correlated moderately with the extent of coronary disease assessed by the SYNTAX score, and they could predict obstructive CAD but not severe disease. 
Depending on coronary angiography, the SYNTAX score is widely applied in evaluating the severity of ACS and predicting outcomes (8). Compared with the SYNTAX score, the thrombolysis in myocardial infarction (TIMI) risk index is a far simpler tool (9). Calculated using only the age, heart rate and systolic blood pressure (SBP), the TIMI risk index has been used to predict mortality in patients with STEMI (10). Pieces of evidence indicated that the TIMI risk index holds value in predicting early mortality of patients with STEMI (9). However, the value of the TIMI risk index in long-term outcome prediction has rarely been investigated, particularly for patients with multiple vessel disease. The present study aimed to investigate the efficacy of TIMI risk index in prediciting long-term outcomes of patents with ST-elevation myocardial infarction and multiple vessel disease.

\section{Materials and methods}

Patients. In the present prospective observational cohort study, all patients encountered from Department of Cardiology, Xianyang Hospital of Yan'an University (Shanxi, China) between June 2013 and July 2014 meeting the following criteria were enrolled: i) Age of 18-80 years; ii) examination with digital subtraction angiography and diagnosis of STEMI and multiple vessel disease; iii) revascularization treatment of percutaneous coronary intervention (PCI); iv) survival of the patient in the hospital; v) the patient and family must be willing to participate and signed informed consent. The exclusion criteria were as follows: i) Patients diagnosed with non-(N)STEMI or unstable angina; ii) patients in a coma or condition of unconsciousness; iii) patients and family do not cooperate with the follow-up. According to the 2017 ESC Guidelines for the Management of STEMI, STEMI was defined as electrocardiographic ST-segment elevation of $\geq 2 \mathrm{~mm}$ in 2 or more contiguous chest leads or $\geq 1 \mathrm{~mm}$ in 2 or more limb leads or new onset of left bundle-branch block, together with chest pain or other typical symptoms and elevated troponin levels $>99$ th percentile [Immunoscattering turbidimetric method; cardiac troponin $\mathrm{T}(\mathrm{cTnT})<0.5 \mathrm{mg} / 1$, cardiac troponin $\mathrm{I}<0.03 \mathrm{mg} / \mathrm{l}]$ (11). A flow chart depicting the study design is presented in Fig. 1.

A total of 428 patients with STEMI were enrolled in the present prospective observational cohort study. All patients and their family were informed of the present study and provided written informed consent. The present study was approved by the Medical Ethics Committee of Xianyang Hospital of Yan'an University (Xianyang, China).

PCI procedure and treatment. Coronary angiography and interventional therapy were performed using conventional methods. PCI strategy and stent selection were determined by the surgeon according to the condition of each patient. PCI was successfully defined as coronary angiography exhibiting residual lumen stenosis $<10 \%$ and blood flow classification was TIMI grade 3. In patients with emergency PCI, aspirin (300 mg) was administered as soon as possible prior to surgery and oral clopidogrel (300-600 mg) or (ticagrelor $180 \mathrm{mg}$ ) was orally administered. Patients with elective PCI who had no contraindications and no history of long-term oral anti-platelet drug treatment prior to surgery received oral aspirin (300 mg) and clopidogrel $(300 \mathrm{mg}$ ) or ticagrelor $(180 \mathrm{mg})$ at least $24 \mathrm{~h}$ prior to surgery. Patients were given oral aspirin (100 mg/day) and clopidogrel (75 mg/day) or telgrelor (90 $\mathrm{mg}$ twice daily) for at least one year after surgery and oral secondary prevention drugs according to the guidelines.

Group and data collection. Clinical data were collected from the electronic database of the hospital. Routine myocardial injury markers, including cardiac troponin $\mathrm{T}$ (cTnT), as well as creatine kinase-myocardial band and other laboratory indicators, including creatinine, NT-proB-type natriuretic peptide and high-sensitivity C-reactive protein (hsCRP), were also tested and recorded. The TIMI risk index was defined as follows: [Heart rate $\left.\mathrm{x}(\mathrm{age} / 10)^{2}\right] / \mathrm{SBP}$ (heart rate measured in bpm; age measured in years and SBP measured in $\mathrm{mmHg}$ ). Heart rate, age and SBP were the values on admission. The SYNTAX score was calculated with all required parameters at admission via the website (http://www.syntaxscore.com/). The required coronary angiography results for the SYNTAX score were confirmed by at least two cardiologists or radiologists (12). Receiver operating characteristic (ROC) curve analysis was performed to determine the value of the TIMI risk index in predicting all-cause death and the best cut-off value was determined to be 30.35. The enrolled patients were divided into the high TIMI group (>30.35) and low TIMI group (<30.35).

Clinical endpoints. The primary endpoint was defined as all-cause mortality during the follow-up, while the secondary endpoints were considered to be a composite of adverse events, including myocardial infarction, stroke, unscheduled revascularization or rehospitalization for heart failure. Myocardial infarction was confirmed using a commonly used criteria (11). Unscheduled revascularization was defined as revascularization treatment of any coronary artery disease driven by ischemic symptoms or events, including PCI and coronary artery bypass grafting.

Follow-up. All enrolled patients were followed up by telephone or visits to the clinic at 1, 3,6 and 12 months every year following discharge for a total of 5 years or until primary or secondary endpoints. Items determined at the follow-up included survival, myocardial infarction, stroke, unscheduled revascularization or rehospitalization due to heart failure. Data for patients with self-dropout or missed contact were presented as censored data, which was included in the survival analysis.

Statistical analysis. SPSS version 19.0 (IBM Corp.) was used for statistical analysis in the present study. Kolmogorov-Smirnov (K-S) test was performed to assess the normality distribution of variables. Continuous variables following a normal distribution were expressed as the mean \pm standard deviation and categorical variables were presented as proportions, while continuous variables not fitting the normal distribution were described as the median and interquartile range. Comparison of continuous variables between different groups was performed using one-way analysis of variance and Tukey's multiple-comparisons test of independent samples. The 


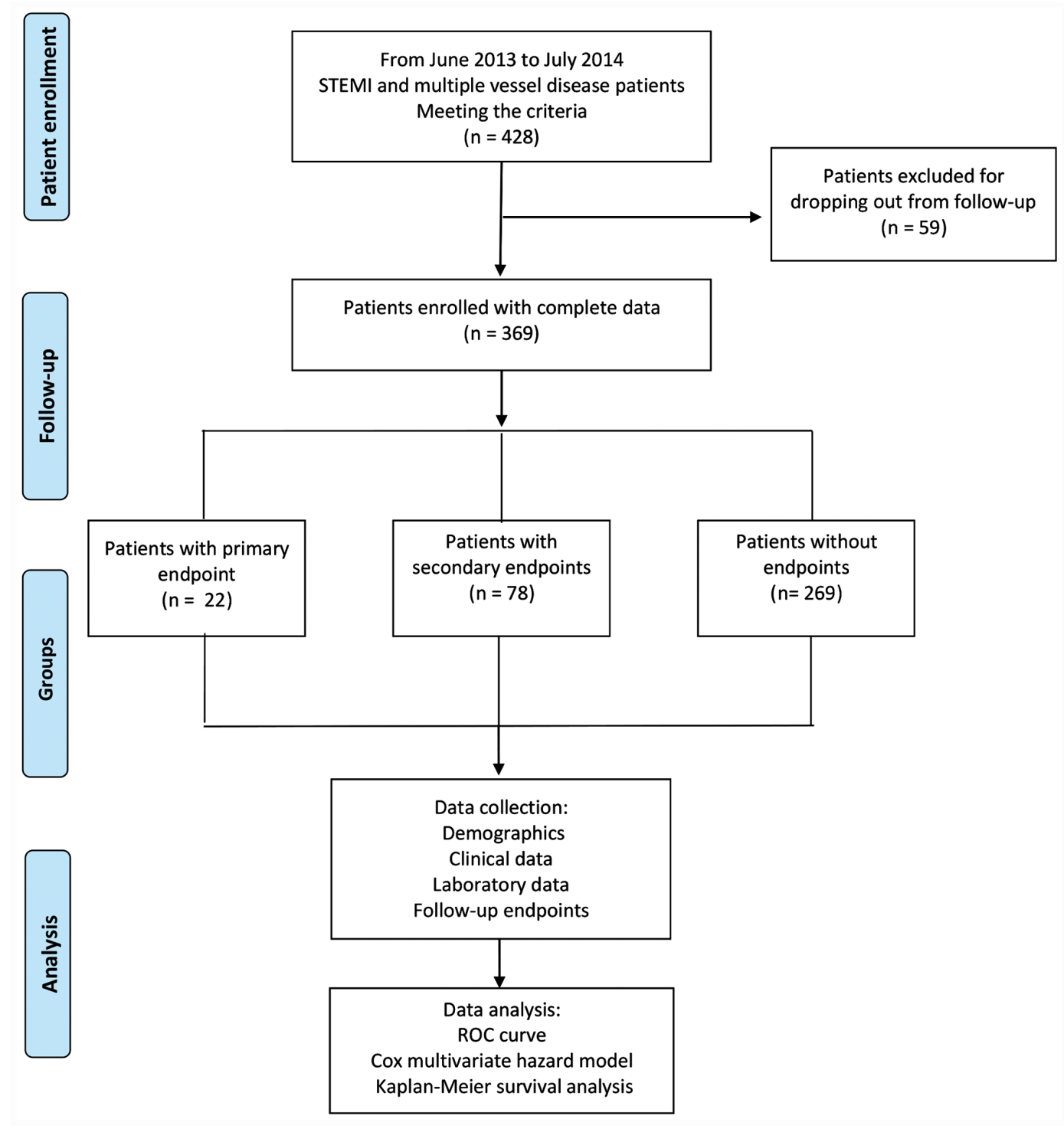

Figure 1. Flow chart of study design. STEMI, ST-elevation myocardial infarction; ROC, receiver operating characteristic.

Kruskal-Wallis test and Dunn's multiple-comparisons test were adopted for comparison between different groups for non-normal variables of independent samples. The $\chi^{2}$ test was performed for comparison of categorical variables.

The Cox hazards model was adopted as the regression method to compare the relative hazard between the high TIMI group and the low TIMI group. Univariate analysis between covariates and endpoints was performed and covariates with $\mathrm{P}<0.10$ were also subjected to multivariate analysis. Kaplan-Meier survival curves and log-rank tests were used to compare the survival status between subgroups. Receiver Operating Characteristic (ROC) analysis was conducted to determine the cut-off value of TIMI index and SYNTAX score for predicting long-term mortality, where the area under curve (AUC) was also calculated. $\mathrm{P}<0.05$ was considered to indicate statistical significance.

\section{Results}

Demographics and clinical data.Among all 428 patients enrolled in the present study, 369 patients completed the follow-up and passed the final data integrity check after five years of follow-up. The average follow-up time was $52.3 \pm 18.4$ months. During the follow-up, 22 patients died (6.0\%), while 78 patients developed secondary endpoints (21.1\%). The demographics and clinical data of patients with or without endpoints are compared in Table I. Patients with primary endpoint were significantly older compared with patients with secondary endpoint $(\mathrm{P}=0.034)$. Patients with secondary endpoint were also significantly older compared with patients without endpoints $(\mathrm{P}=0.020)$. The BMI of patients with secondary endpoint was lower compared with patient from the other two groups $(\mathrm{P}=0.029$ vs. patients without endpoints; $\mathrm{P}=0.013$ vs. patients with primary endpoint), whilst the BMI of patients from other two groups were comparable $(\mathrm{P}=0.342)$. However, there was no statistical difference among the three groups in terms of sex $(\mathrm{P}=0.096)$ and smoking status $(\mathrm{P}=0.882)$. In terms of NYHA grade, patients with primary endpoint was significantly associated with higher NYHA grade compared with those from other two groups (both $\mathrm{P}<0.05$ ), with no statistical difference observed between patients with secondary endpoint and patients without endpoints $(\mathrm{P}=0.908)$. Patients with primary endpoint also had significantly higher 
Table I. Demographic characteristics and clinical data of the patients enrolled stratified based on the availability of data on endpoints.

\begin{tabular}{|c|c|c|c|c|}
\hline Variables & $\begin{array}{l}\text { Patients without } \\
\text { endpoints }(n=269)\end{array}$ & $\begin{array}{l}\text { Patients with the } \\
\text { primary endpoint }(n=22)\end{array}$ & $\begin{array}{l}\text { Patients with the } \\
\text { secondary endpoint }(n=78)\end{array}$ & P-value \\
\hline \multicolumn{5}{|l|}{ Demographics } \\
\hline Age (years) & $62.2 \pm 16.0$ & $74.2 \pm 10.8^{\mathrm{a}}$ & $66.7 \pm 10.0^{\mathrm{a}, \mathrm{b}}$ & $<0.001$ \\
\hline Male sex & $162(60.2)$ & $17(77.3)$ & $55(70.5)$ & 0.096 \\
\hline BMI $\left(\mathrm{kg} / \mathrm{m}^{2}\right)$ & $24.9 \pm 3.2$ & $25.6 \pm 2.7$ & $23.9 \pm 3.1^{\mathrm{a}, \mathrm{b}}$ & 0.021 \\
\hline Smoking & $98(36.4)$ & $9(40.9)$ & $30(38.5)$ & 0.882 \\
\hline NYHA grade & & & & 0.011 \\
\hline I & $72(26.8)$ & $3(13.6)$ & $17(21.8)$ & \\
\hline II & $87(32.3)$ & $6(27.3)$ & $31(39.7)$ & \\
\hline III & $90(33.5)$ & $6(27.3)$ & $22(28.2)$ & \\
\hline IV & $20(7.4)$ & $7(31.8)^{\mathrm{a}}$ & $8(10.3)^{b}$ & \\
\hline LVEF & $49.6 \pm 10.0$ & $43.6 \pm 12.6^{\mathrm{a}}$ & $49.8 \pm 9.4^{\mathrm{b}}$ & 0.025 \\
\hline \multicolumn{5}{|l|}{ Comorbidities } \\
\hline Heart failure & $69(25.7)$ & $10(45.5)$ & $20(25.6)$ & 0.127 \\
\hline Hypertension & $64(23.8)$ & $12(54.5)^{\mathrm{a}}$ & $23(29.5)^{b}$ & 0.006 \\
\hline Diabetes mellitus & $37(13.8)$ & $4(18.2)$ & $11(14.1)$ & 0.848 \\
\hline Chronic kidney disease & $13(4.8)$ & $2(9.1)$ & $4(5.1)$ & 0.686 \\
\hline Chronic lung disease & $35(13.0)$ & $3(13.6)$ & $5(6.4)$ & 0.266 \\
\hline Cerebrovascular disease & $18(6.7)$ & $1(4.5)$ & $5(6.4)$ & 0.925 \\
\hline Tumor & $7(2.6)$ & $1(4.5)$ & $2(2.6)$ & 0.861 \\
\hline \multicolumn{5}{|l|}{ Laboratory test at admission } \\
\hline Peak cTnT (ng/dl) & $4.87(3.16,6.44)$ & $6.31(3.85,6.99)$ & $4.14(2.74,5.99)^{\mathrm{b}}$ & 0.019 \\
\hline Peak CK-MB (ng/dl) & $549.3(420.6,671.4)$ & $466.3(387.0,663.1)$ & $603.2(408.2,713.5)$ & 0.299 \\
\hline Creatinine $(\mu \mathrm{mol} / \mathrm{l})$ & $104(75,132.5)$ & $105(74.25,136)$ & $92(69.75,127)$ & 0.278 \\
\hline NT-proBNP (ng/l) & $71.1(27.25,123.1)$ & $91.3(55.73,168.43)$ & $74.2(35.88,123.15)$ & 0.212 \\
\hline $\operatorname{hsCRP}(\mathrm{mg} / \mathrm{l})$ & $21.5(12.95,29.5)$ & $30.9(24.7,42.65)^{\mathrm{a}}$ & $26.25(17.5,35.08)^{\mathrm{a}, \mathrm{b}}$ & $<0.001$ \\
\hline SYNTAX score & $24.4(18.9,30.35)$ & $29.9(25.05,38.18)^{\mathrm{a}}$ & $27.65(23.88,31.2)^{\mathrm{a}, \mathrm{b}}$ & $<0.001$ \\
\hline TIMI risk index & $13(8.5,16)$ & $17.5(13.5,23.25)^{\mathrm{a}}$ & $17.5(12,22)^{\mathrm{a}}$ & $<0.001$ \\
\hline
\end{tabular}

Values are expressed as the mean \pm standard deviation, $\mathrm{n}(\%)$ or the median (lower quartile, higher quartile). ${ }^{\mathrm{a} P}<0.05$ vs. patients without endpoints and ${ }^{\mathrm{b}} \mathrm{P}<0.05$ vs. Patients with the primary endpoint. BMI, body mass index; NYHA grade, New York Heart Association class; LVEF, left ventricular ejection fraction; cTnT, cardiac troponin T; CK-MB, creatine kinase-myocardial band; NT-proBNP, NT-proB-type natriuretic peptide; hsCRP, high-sensitivity C-reactive protein; TIMI, thrombolysis in myocardial infarction.

proportions of hypertension compared with the other two groups $(\mathrm{P}=0.006)$, while there was no significant difference all for other comorbidities recorded (All P>0.05). Patients with secondary endpoints had lower Peak cTnT levels compared with those with primary endpoint $(\mathrm{P}=0.007)$, but no statistical differences were found with the other two group comparisons $(\mathrm{P}>0.05)$. With regards to the SYNTAX score, patients with primary endpoint was higher compared with those in the other two groups $(\mathrm{P}<0.05)$, whilst patients with second endpoint was higher than those without endpoint $(\mathrm{P}<0.05)$. However, TIMI risk index of patients with no endpoint was lower compared with patients with primary endpoints and patients with secondary endpoints $(\mathrm{P}<0.001)$, but there was no difference between those from primary and secondary endpoints $(\mathrm{P}=0.399)$.

ROC analysis. Fig. 2 presents the ROC analysis for the TIMI risk index to predict all-cause death. ROC analysis demonstrated that the TIMI risk index was a significant predictive factor with an area under the curve (AUC) of 0.705 (95\% CI: 0.591-0.819, $\mathrm{P}=0.001)$. As for the SYNTAX score, there was no statistical significance for the prediction of all-cause death [AUC $=0.559$, 95\% CI: 0.432-0.687, $\mathrm{P}=0.350$; Fig. 3].

Cox regression analysis. Cox regression analysis was performed to evaluate the risk factors for the primary and secondary endpoints. As presented in Table II, multivariate Cox regression analysis demonstrated that a high TIMI risk index, age and hypertension were independent risk factors for all-cause death (all $\mathrm{P}<0.05)$. Patients with a high TIMI risk index had a hazard ratio of 3.709 (95\% CI: 1.521-9.046) for all-cause death compared to those with a low TIMI risk index $(\mathrm{P}=0.004)$.

The risk factors for secondary endpoints were also explored (Table III). The Cox regression analysis, whether adjusted or unadjusted, indicated that a high TIMI risk index was not 
Table II. Multivariate Cox regression analysis of primary endpoints.

\begin{tabular}{|c|c|c|c|c|c|c|}
\hline Risk factor & $\mathrm{B}$ & SE & Wald & P-value & HR & $95 \% \mathrm{CI}$ \\
\hline TIMI risk index & 1.311 & 0.455 & 8.304 & 0.004 & 3.709 & $1.521-9.046$ \\
\hline Age & 0.050 & 0.018 & 7.230 & 0.007 & 1.051 & $1.014-1.089$ \\
\hline BMI & 0.080 & 0.076 & 1.093 & 0.296 & 1.083 & $0.933-1.258$ \\
\hline NYHA grade & 0.431 & 0.244 & 3.115 & 0.078 & 1.539 & $0.953-2.485$ \\
\hline Hypertension & 1.524 & 0.471 & 10.463 & 0.001 & 4.589 & $1.823-11.552$ \\
\hline cTNT & 0.147 & 0.126 & 1.375 & 0.241 & 1.159 & $0.906-1.482$ \\
\hline hsCRP & 0.089 & 0.022 & 15.638 & 0.999 & 1.000 & $1.046-1.093$ \\
\hline
\end{tabular}

BMI, body mass index; NYHA, New York Heart Association; cTnT, cardiac troponin T; hsCRP, high-sensitivity C-reactive protein; HR, heart rate; SE, standard error; TIMI, thrombolysis in myocardial infarction.

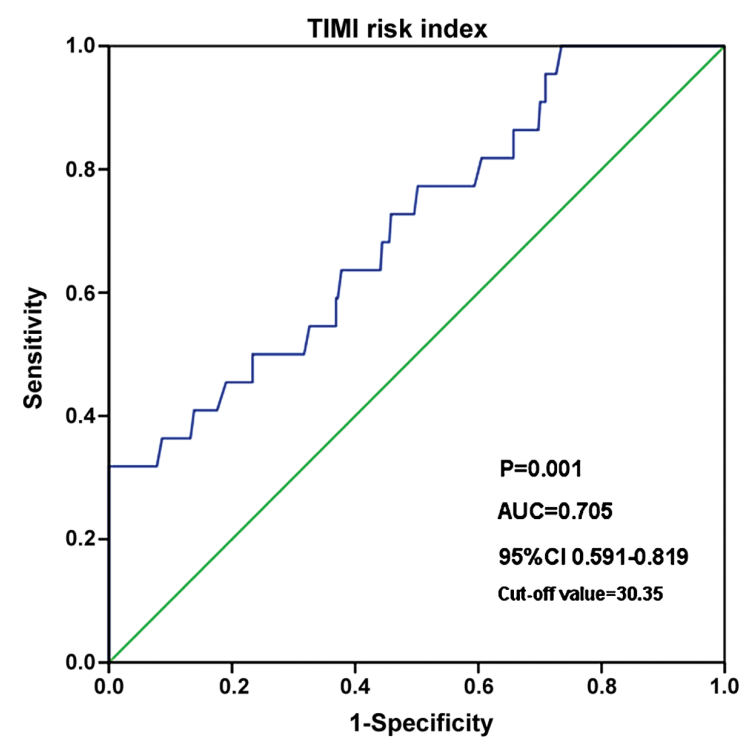

Figure 2. ROC curve for the TIMI risk index to predict long-term mortality. TIMI, thrombolysis in myocardial infarction; ROC, receiver operating characteristic; AUC, area under the ROC curve.

an independent risk factor for the composite of secondary endpoints and also not for each single endpoint (all $\mathrm{P}>0.05$ ).

Survival analysis. To validate the long-term outcomes of the two groups, a survival analysis was performed (Figs. 4 and 5). As presented in Fig. 4, patients with a high TIMI risk index and patients with a low TIMI risk index had significantly different survival curves. The high TIMI group had a significantly lower five-year survival $(\mathrm{P}=0.009)$. However, there was no significant difference between the high TIMI risk index and low TIMI risk index groups in the curves for the incidence of secondary endpoints $(\mathrm{P}=0.527)$.

\section{Discussion}

In the present study, the predictive value of the TIMI risk index regarding the long-term outcomes of patients with STEMI with multiple vessel disease was investigated. The ROC curve analysis suggested that the TIMI risk index was able to predict all-cause mortality within five years after

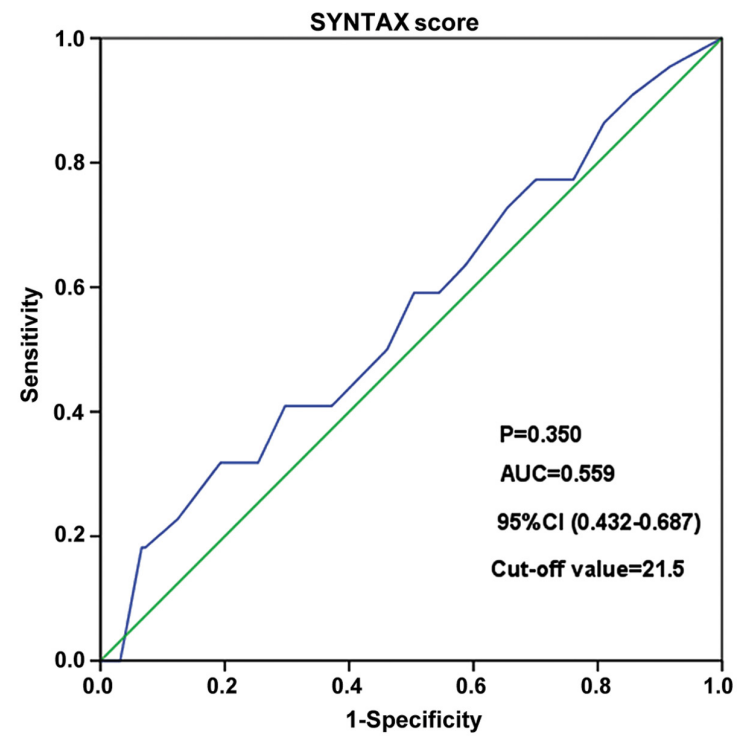

Figure 3. ROC curve for the SYNTAX score to predict long-term mortality. ROC, receiver operating characteristic; AUC, area under the ROC curve.

PCI. Further analysis validated this result by comparing the long-term outcomes for patients with a high TIMI risk index and with a low TIMI risk index, revealing a significant difference in all-cause mortality. Risk factor analysis performed using the Cox regression model proved that a high TIMI risk index (>30.35) was an independent risk factor for all-cause death, but not for any secondary adverse events, including myocardial infarction, stroke, unscheduled revascularization or rehospitalization for heart failure.

Patients with STEMI have a poor prognosis and early identification of high-risk patients is critical, particularly for those with multiple vessel disease (2). A simple, cost-effective and sufficient risk assessment approach helps clinicians take more aggressive interventions and follow-up measures to improve outcomes. The Global Registry of Acute Coronary Events (GRACE) scores is widely used in the clinic for risk stratification of patients with ACS, but these scoring systems require a more detailed medical history, laboratory tests and even hemodynamic parameters to increase the capacity of risk stratification (6). Therefore, these scoring systems may have limitations in clinical applications. The TIMI risk index is 
Table III. Multivariate Cox regression analysis of secondary endpoints influenced by high TIMI vs. low TIMI.

\begin{tabular}{|c|c|c|c|c|}
\hline \multirow[b]{2}{*}{ Secondary endpoint } & \multicolumn{2}{|c|}{ Unadjusted } & \multicolumn{2}{|c|}{ Adjusted $^{\mathrm{a}}$} \\
\hline & HR $(95 \% \mathrm{CI})$ & P-value & $\mathrm{HR}(95 \% \mathrm{CI})$ & P-value \\
\hline Myocardial infarction & $1.336(0.694-2.570)$ & 0.386 & $1.462(0.756-2.830)$ & 0.259 \\
\hline Stroke & $1.991(0.333-11.916)$ & 0.451 & $2.457(0.386-15.632)$ & 0.341 \\
\hline Emergent revascularization & $0.473(0.140-1.600)$ & 0.487 & $1.413(0.143-1.657)$ & 0.249 \\
\hline Readmission due to heart failure & $2.411(0.647-8.979)$ & 0.190 & $2.333(0.616-8.836)$ & 0.213 \\
\hline Overall & $1.174(0.717-1.923)$ & 0.523 & $1.267(0.770-2.082)$ & 0.352 \\
\hline
\end{tabular}

${ }^{a} \mathrm{HR}$ adjusted by covariates of age, body mass index, New York Heart Association, hypertension, cardiac troponin T and high-sensitivity C-reactive protein. HR, hazard ratio; TIMI, thrombolysis in myocardial infarction.

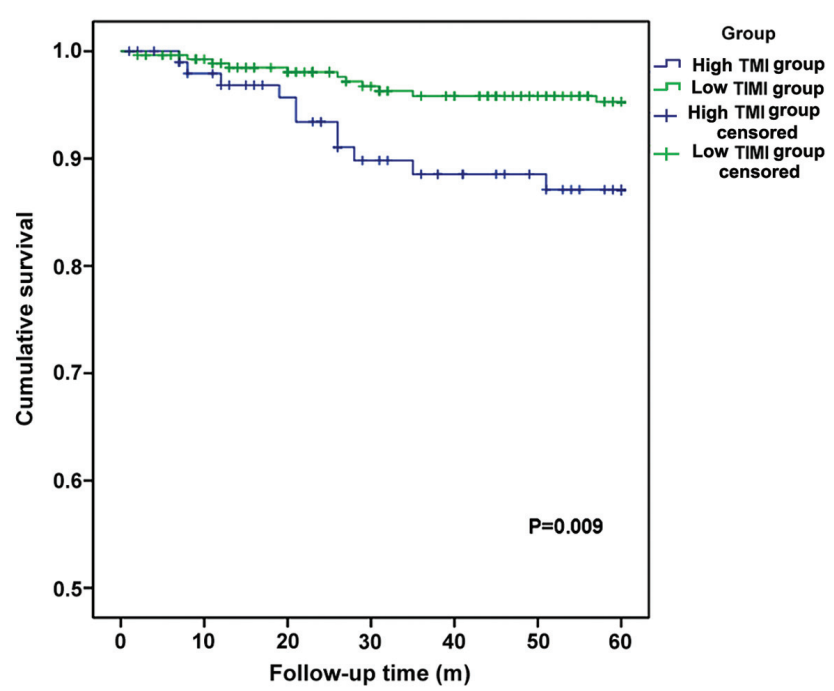

Figure 4. Kaplan-Meier survival curves for the primary endpoint (overall survival) for the high TIMI group and low TIMI group. TIMI, thrombolysis in myocardial infarction.

calculated using three parameters: Age, heart rate and systolic blood pressure, which is convenient for clinicians to rapidly determine the condition of the patient during the first medical consultation and provide a necessary strategy for subsequent risk stratification, including the accurate GRACE score (13). Based on the TIMI risk index, the clinician may simply classify the patient as high- or low-risk regarding long-term mortality and may then adopt different treatment strategies.

Plenty of studies have suggested the value of the TIMI risk index in risk stratification of patients with ACS. The InTIME II substudy demonstrated that the TIMI risk index was useful in the rapid triage of patients with STEMI outside the hospital or on first arrival at the hospital as a simple tool and may predict in-hospital mortality (14). The Effective Cardiac Treatment study indicated that the TIMI risk index is a simple, valid and moderately accurate tool for risk stratification for early death in patients with STEMI and NSTEMI in a community setting (9). In the TIMI 2 clinical trial, the TIMI risk index was demonstrated to predict long-term mortality and chronic heart failure (CHF) as well as composite death and CHF in patients with STEMI (15). However, most studies focused on the value of the TIMI risk index in the short term rather than long-term

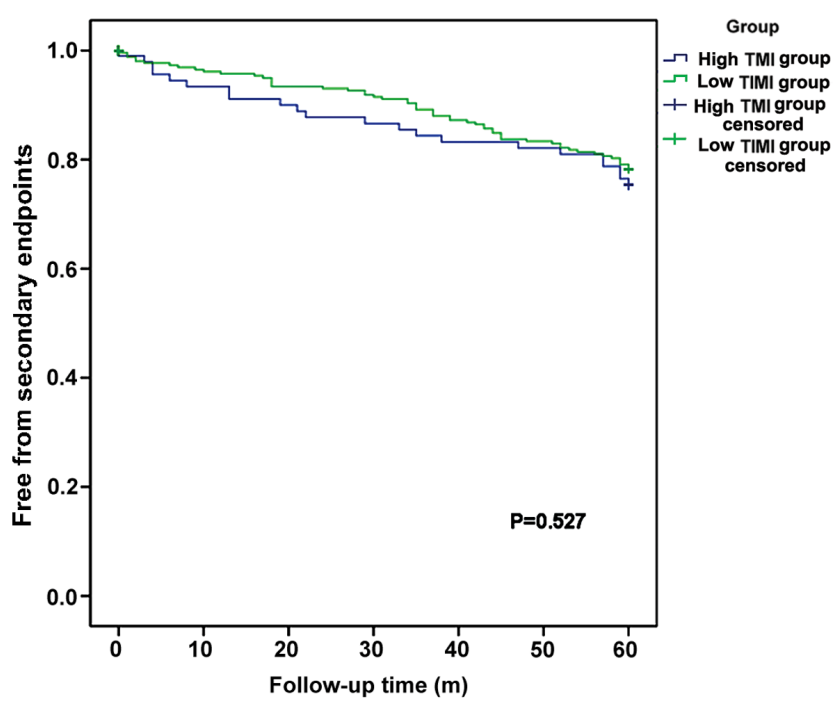

Figure 5. Kaplan-Meier survival curve for secondary endpoints, including myocardial infarction, stroke, unscheduled revascularization or rehospitalization due to heart failure for the high TIMI group and low TIMI group. TIMI, thrombolysis in myocardial infarction.

outcomes. Of note, the revascularization rates of those studies were $53-36 \%(9,10,16)$, not as high as those in recent studies.

At present, there is limited evidence regarding the predictive value of the TIMI risk index in STEMI patients with multiple vessel disease. The present study demonstrated that the TIMI risk index is of value in predicting long-term mortality of patients with STEMI and multiple vessel disease. Multivariate Cox regression analysis revealed that age, hypertension and the TIMI risk index were independent risk factors for the primary endpoint. As mentioned above, the TIMI risk index was calculated using the age, heart rate and SBP. Hence, the TIMI risk index is associated with age and hypertension. However, the TIMI risk index is a comprehensive indicator reflecting the condition of the patient. The regression model also demonstrated its independence from age and hypertension.

Although the TIMI risk index was associated with the primary endpoint, it had no predictive value regarding secondary endpoints. This may be due to two reasons: On the one hand, the sample size may have been insufficient to obtain a significant difference, even regarding individual adverse 
events, including heart failure. On the other hand, the TIMI risk index was not associated with those adverse events, but with other possibly lethal complications.

Comparison of the SYNTAX score and TIMI risk index indicated that the SYNTAX score had a lower predictive value than the TIMI risk index. Brkovic (17) compared the prognostic value of the SYNTAX, GR ACE, TIMI,Zwolle risk score (ZRS), the Controlled Abciximab and Device Investigation to Lower Late Angioplasty Complications (CADILLAC) and Primary Angioplasty in Myocardial Infarction (PAMI) risk scores in patients with acute STEMI treated by primary percutaneous coronary intervention and indicated that the SYNTAX score improves the prognostic performance of the well-established GRACE, TIMI, ZRS and PAMI clinical scores, but not the CADILLAC risk score. A Chinese study suggested that TIMI risk index (TRI) is independently associated with a SYNTAX score of $\geq 33$. TRI is also an independent risk factor for 2-year all-cause death, cardiac death and stent thrombosis in patients with AMI undergoing PCI (16). The cut-off value for the TIMI risk index in the present study was 30.35 which was almost the first-quartile level, with 100 patients $(27.1 \%)$ above and 2.69 patients $(72.9 \%)$ below this cut-off value. The proportion of patients with a high TIMI risk index in the group of patients with the primary endpoint was higher than that in the other two groups. This cut-off value was higher than those in previous studies (16), with a cut-off value of 23.05, which may be attributed to different follow-up times and inclusion criteria. The sample size and observational nature of the study were the limitations of the present study, and further cohort studies and meta-analyses are required to validate the present results.

In conclusion, the TIMI risk index was associated with long-term mortality of patients with STEMI and multiple vessel disease, and may be utilized for risk stratification for such patients.

\section{Acknowledgements}

Not applicable.

\section{Funding}

No funding was received.

\section{Availability of data and materials}

The datasets used and/or analyzed during the current study are available from the corresponding author on reasonable request.

\section{Authors' contributions}

XF and ZL designed and led this study. ML and JC collected and analyzed the general patient data. XF and ML wrote the manuscript. XF and ZL confirm the authenticity of all the raw data. All authors read and approved the final manuscript.

\section{Ethics approval and consent to participate}

This study was approved by the Medical Ethics Committee of Yueqing Third People's Hospital (Yueqing, China). The patients provided written informed consent regarding the use of their data.

\section{Patient consent for publication}

Not applicable.

\section{Competing interests}

The authors declare that they have no competing interests.

\section{References}

1. Ruff CT and Braunwald E: The evolving epidemiology of acute coronary syndromes. Nat Rev Cardiol 8: 140-147, 2011.

2. Lee WC, Wu BJ, Fang CY, Chen CJ, Yang CH, Yip HK, Hang CL, Wu CJ and Fang HY: Timing of staged percutaneous coronary intervention for a non-culprit lesion in patients with anterior wall ST segment elevation myocardial infarction with multiple vessel disease. Int Heart J 57: 417-423, 2016.

3. Acet H, Ertas F, Bilik MZ, Aydın M, Yüksel M, Polat N, Yıldız A, Özyurtlu F, Akıl MA, Çiftçi L, et al: The relationship of TIMI risk index with SYNTAX and Gensini risk scores in predicting the extent and severity of coronary artery disease in patients with STEMI undergoing primary percutaneous coronary intervention. Ther Adv Cardiovasc Dis 9: 257-266, 2015.

4. Damman P, Kampinga MA, van der Horst IC, Woudstra P, Grundeken MJ, Kuijt WJ, Harskamp RE, Nijsten MW, Zijlstra F, Tijssen JG, et al: Multiple biomarkers for the prediction of short and long-term mortality after ST-segment elevation myocardial infarction: The Amsterdam Groningen collaboration. J Thromb Thrombolysis 36: 42-46, 2013.

5. He J, Li J, Wang Y, Hao P and Hua Q: Neutrophil-to-lymphocyte ratio (NLR) predicts mortality and adverse-outcomes after ST-segment elevation myocardial infarction in Chinese people. Int J Clin Exp Pathol 7: 4045-4056, 2014.

6. Sakamoto JT, Liu N, Koh ZX, Fung NX, Heldeweg ML, Ng JC and Ong ME: Comparing HEART, TIMI, and GRACE scores for prediction of 30-day major adverse cardiac events in high acuity chest pain patients in the emergency department. Int J Cardiol 221: 759-764, 2016.

7. Hammami R, Jdidi J, Mroua F, Kallel R, Hentati M, Abid L and Kammoun S: Accuracy of the TIMI and GRACE scores in predicting coronary disease in patients with non-STelevation acute coronary syndrome. Rev Port Cardiol 37: 41-49, 2018.

8. Tarasov RS, Ganiukov VI, Shilov AA, Barbarash OL and Barbarash LS: Prognostic value of SYNTAX score for outcomes and revascularization strategy choice in ST-segment elevation myocardial infarction patients with multivessel coronary artery disease. Ter Arkh 84: 17-21, 2012 (In Russian).

9. Bradshaw PJ, Ko DT, Newman AM, Donovan LR and Tu JV: Validation of the Thrombolysis In Myocardial Infarction (TIMI) risk index for predicting early mortality in a population-based cohort of STEMI and non-STEMI patients. Can J Cardiol 23: 51-56, 2007.

10. WiviottSD, Morrow DA, Frederick PD, Giugliano RP, Gibson CM, McCabe $\mathrm{CH}$, Cannon CP, Antman EM and Braunwald E: Performance of the thrombolysis in myocardial infarction risk index in the National Registry of Myocardial Infarction-3 and -4: A simple index that predicts mortality in ST-segment elevation myocardial infarction. J Am Coll Cardiol 44: 783-789, 2004.

11. Ibanez B, James S, Agewall S, Antunes MJ, Bucciarelli-Ducci C, Bueno H, Caforio ALP, Crea F, Goudevenos JA, Halvorsen S, et al: 2017 ESC Guidelines for the management of acute myocardial infarction in patients presenting with ST-segment elevation: The Task Force for the management of acute myocardial infarction in patients presenting with ST-segment elevation of the European Society of Cardiology (ESC). Eur Heart J 39: 119-177, 2018.

12. Karabag Y, Çağdaş M, Rencuzogullari I, Karakoyun S, Artaç İ, İliş D, Yesin M, Öterkus M, Gokdeniz T, Burak C and Tanboğa IH: Comparison of SYNTAX score II efficacy with SYNTAX score and TIMI risk score for predicting in-hospital and long-term mortality in patients with ST segment elevation myocardial infarction. Int J Cardiovasc Imaging 34: 1165-1175, 2018. 
13. Jakimov T, Mrdovic I, Filipovic B, Zdravković M, Djoković A, Hinić S, Milić N and Filipović B: Comparison of RISK-PCI, GRACE, TIMI risk scores for prediction of major adverse cardiac events in patients with acute coronary syndrome. Croat Med J 58: 406-415, 2017.

14. Morrow DA, Antman EM, Giugliano RP, Cairns R Charlesworth A, Murphy SA, de Lemos JA, McCabe CH and Braunwald E: A simple risk index for rapid initial triage of patients with ST-elevation myocardial infarction: An InTIME II substudy. Lancet 358: 1571-1575, 2001.

15. Truong QA, Cannon CP, Zakai NA, Rogers IS, Giugliano RP, Wiviott SD, McCabe CH, Morrow DA and Braunwald E: Thrombolysis in myocardial Infarction (TIMI) risk index predicts long-term mortality and heart failure in patients with ST-elevation myocardial infarction in the TIMI 2 clinical trial. Am Heart J 157: 673-679.e1, 2009.

16. Chen Y, Song Y, Xu JJ, Tang XF, Wang HH, Jiang P, Jiang L, Liu R, Zhao XY, Gao LJ, et al: Relationship between thrombolysis in myocardial infarction risk index and the severity of coronary artery lesions and long-term outcome in acute myocardial infarction patients undergoing percutaneous coronary intervention. Zhonghua Xin Xue Guan Bing Za Zhi 46: 874-881, 2018 (In Chinese).
17. Brkovic V, Dobric M, Beleslin B, Giga V, Vukcevic V, Stojkovic S, Stankovic G, Nedeljkovic MA, Orlic D, Tomasevic M, et al: Additive prognostic value of the SYNTAX score over GRACE, TIMI, ZWOLLE, CADILLAC and PAMI risk scores in patients with acute ST-segment elevation myocardial infarction treated by primary percutaneous coronary intervention. Int J Cardiovasc Imaging 29: 1215-1228, 2013.

c) (i) $९$ This work is licensed under a Creative Commons Cy NO AD Attribution-NonCommercial-NoDerivatives 4.0 International (CC BY-NC-ND 4.0) License. 